\title{
Ambiente domiciliar e alterações do desenvolvimento em crianças de comunidade da periferia de São Luís - M A
}

\author{
Home environment and alterations in the development \\ of children in a community of the outskirts of São Luís - M A
}

Fernando Lamy Filho ${ }^{1}$

Sandra M aria de M edeiros ${ }^{2}$

Zeni Carvalho Lamy ${ }^{1}$

M aria Elizabeth Lopes M oreira ${ }^{3}$

${ }^{1}$ Departamento de M edicina III, Centro de Ciências da Saúde, UniversidadeFederal do M aranhão. Rua Silva Jardim 215/Co - Hospital Universitário-Unidade $M$ aterno Infantil

Centro. 65021-000 São Luís MA.

lamyfilho@gmail.com

${ }^{2}$ FaculdadeSanta Terezinha,

CEST

${ }^{3}$ Instituto Fernandes

Figueira, Fiocruz, RJ
Abstract Themain bonds of infantsin early childhood are established in the home environment. The home is fundamental for providing stimuli that can influence the development of the child. A cross-sectional study was conducted in a low income community in the outskirts of the city of São Luís to analyze the provision of stimuli in the home environment of 2-year-old children and the potential association with retardation in child development. A random sample of 1762 -year-old children registered in community outpatient healthcare departments was assessed. Two validated instruments were used, namely the Home O bservation for $M$ easurement of the Environment (H OM E) Inventory and the Gesell M easurement of Development Scale. Data were gathered in the homes. Logistic regressions were carried out taking the Home Inventory (low or average/high) and the Gesell Scale (suspicion of delay/normal) as outcome variables. Low family income, low maternal and paternal schooling, number of residents, number of children $<5$ years old and small number of rooms in the house were considered risk factors for low H ome scores, which were associated with the suspicion of development delay. The quality and quantity of environmental stimuli in the family context proved to be essential for the development of the children evaluated.

Key words Home environment, Child development
Resumo Na primeira infância, os principais vínculos da criança são estabelecidos no ambiente doméstico. Esteéfundamental quanto à oferta de estímulos que podem influenciar seu desenvolvimento. Com o objetivo de analisar o ambiente domiciliar de crianças de 2 anos de idade, quanto à oferta de estímulos eà sua relação com suspeitas de atrasos de desenvolvimento, realizou-se estudo transversal, em comunidade de baixa renda na periferia de São Luis. Calculou-se amostra aleatória de 176 crianças de 2 anos de idadecadastradas em unidades de saúde da comunidade. Utilizaram-se dois instrumentos validados: o Inventory $\mathrm{H}$ ome (medida de estímulos domiciliares) e a Gesell Scale (medida de desenvolvimento). Os dados foram col etados nas próprias casas. Foram realizadas regressões logísticas tendo como variáveis resposta o HOME (baixo ou médio/alto) e Gesell (com suspeita deatraso ou s/atraso). Renda familiar baixa, baixa escolaridade materna e paterna, $\mathrm{n}$ - de residentes, $\mathrm{n}$ - de filhos $<5$ anos e baixo $\mathrm{n}$ - de cômodos foram fatores de risco para HOM E baixo. Este, por sua vez, associou-se com a suspeita de atraso no desenvolvimento (Gesell baixo). A qualidade e a quantidade de estímulos ambientais presentes no contexto familiar mostraram-se fundamentais para o desenvolvimento global das crianças avaliadas.

Palavras-chave Ambiente doméstico, D esenvolvimento infantil 
Introdução

Os riscos para a ocorrência de atraso no desenvolvimento infantil estão concentrados em três grandes grupos, sendo um deles o risco ambiental. Para um ambiente ser considerado de boa qualidade para o desenvolvimento infantil ele deve oferecer relacionamentos sustentadores ${ }^{1,2}$, promoção da segurança física, proteção contra doenças, suprimento das necessidades básicas, além de experiências diversificadas ${ }^{3}$.

Há várias décadas pesquisas vêm sendo realizadas para identificar quais os aspectos ambientais quemais influenciam o processo de desenvolvimento infantil. Fatores ligados a más condições de vida como a baixa renda familiar, a baixa escolaridade dos pais e o grande número de irmãos, quase sempre presentes na realidade das periferias das grandes cidades brasileiras, são elementos frequentemente citados na literatura ${ }^{4}$ 6 . Em estudo feito em Pelotas, no sul do Brasil, $\mathrm{H}$ alpern et al. ${ }^{7}$ encontraram como fatores de risco para suspeita de atrasos no desenvolvimento de crianças de 1 ano de idade, a pobreza ea existência de mais de três irmãos no mesmo domicílio. Andrade et al. ${ }^{8}$, em dissertação de mestrado em Salvador, Bahia, encontrou resultados similares aos de $\mathrm{H}$ al pern et al. ${ }^{7}$.

Além dessas, outras influências do ambiente sobre o desenvolvimento são relatadas. CappeIlini et al. ${ }^{9}$, em trabalho recente em Florença, I tália, encontrou diferentes graus de desenvolvimento dos pré requisitos funcionais de psicomotricidade entre crianças criadas em áreas rurais e urbanas dessa cidade, submetidas, portanto, a diferentes estímulos. Fluss et al. ${ }^{10}$ demonstraram que a prevalência da leitura pobre em adolescentes de escolas secundárias foi altamente correlacionada com a posição socioeconômica, demonstrada pela área geográfica da escola pesquisada.

Elementos ligados ao meio doméstico podem levar a um sério comprometimento da qualidade do ambiente, alterando potencialmente 0 de senvolvimento infantil. Assim como em outras grandes cidades brasileiras, em São Luís, M aranhão, algumas áreas periféricas abrigam comunidades ondea realidade de carência pode potencialmentelevar à existência significativa de crianças na faixa etária de 0 a 2 anos de idade com atraso no desenvolvimento neuropsicomotor.

Assim, o presente trabal ho sepropôs a verificar a prevalência do diagnostico de atraso no desenvolvimento em crianças de 2 anos de idade em uma comunidade carente em São Luís, e sua relação com elementos do ambiente doméstico.
Acredita-se que o conhecimento desses elementos pode vir a favorecer a aplicação de estratégias efetivas para o controle desse tipo de alterações.

\section{M etodologia}

Foi realizado estudo transversal em que se investigou a associação entre elementos do ambiente domiciliar e o desenvolvimento neuropsicomotor de crianças aos 2 anos de idade, residentes na Vila Cidade O límpica, uma área de ocupação da cidadedeSão Luís, a princípio ilegal, ocorridano início da década de 90, hoje considerada propriedade dos moradores. Esse bairro tem tido crescimento desordenado, possuindo, no momento da coleta de dados, uma população heterogênea, oficial mente estimada em 36.642 habitantes, com grande mobilidade demográfica podendo chegar, na prática, até 56.000 moradores $^{11}$. Não dispõe de um sistema de distribuição de água, não há saneamento básico e o nível de organização comunitário é relativamente baixo.

A população alvo constituiu-se de 2.524 crianças com idade de 1 a 4 anos cadastradas no Programa Saúde da Família da área. Identificaramse, entre essas, 412 com idade entre 2 e 3 anos. Após cálculo no programa EPIINFO 2003, foi escolhida, de forma aleatória, uma amostra de $184(44,6 \%)$ deslas, que compuseram o total de crianças estudadas. Para o cálculo amostral foram utilizados um intervalo de confiança de $95 \%$, com poder de $80 \%$ e probabilidade de erro tipo alfa de $5 \%$. Para controle de confundimento foi utilizada regressão logística do tipo stepwize com ponto de corte de 0,20 no p-valor para entrada no modelo.

A escolha da faixa dos 2 anos deidadeno estudo deveu-se a dois fatos. Em primeiro lugar por ser nesse momento, final do período sensório motor, que a criança entra no período pré-operatório e, portanto, desenvolve habilidades simbólicas e de estruturação do pensamento e da linguagem, ampliando sua relação com o meio e passando a expressar, o que já vinha sendo construído ao longo do primeiro ano ${ }^{12}$. Além disso, entendeu-se que seria mais adequado, do ponto de vista epidemiológico, trabal har-se com um período de idade menor devido à grande variabilidade de fases do desenvolvimento que poderiam estar envolvidas em uma faixa ponderal mais ampla, incluindo a influência do ambiente escolar.

Além da faixa etária, foram adotados como critérios de inclusão: residir na área urbana da comunidadeVila CidadeO límpica e estar cadas- 
trado nas unidades de saúde do bairro. Não foram incluídas crianças com problemas durante a gestação ou parto, infecções congênitas do tipo TORCHS, gemelaridade, baixo peso ao nascer, prematuridade, anomalias cromossômicas, distúrbios neurológicos e desnutrição no primeiro ano de vida.

Antes da coleta dos dados realizou-se o treinamento da equipe para a aplicação do questionário "Home Observation for Measurement of the Environment" (HOME, versão Infant/Todd(er) ${ }^{13} \mathrm{e}$ da aplicação da Escala de Gesell para desenvolvimento infantil ${ }^{14}$. 0 inquérito domiciliar foi aplicado na própria residência da criança assim como a Escala de Gesell. Além dessas duas avaliações, foram coletados dados sobre a situação sócio-econômico-demográfica das famílias entrevistadas.

Antes das entrevistas, foi solicitada a assinatura do Termo de Consentimento Livre e Esclarecido pelos pais ou responsáveis pela criança. Foi obtida também a autorização do Comitê de Ética em Pesquisa do Hospital Universitário da Universidade Federal do M aranhão.

\section{Resultados}

Foram estudadas 176 crianças saudáveis entre dois e três anos completos. Oito pacientes foram perdidos por recusa dos pais ou por dificuldade na local ização do domicílio. A prevalência desuspeita deatraso no desenvolvimento nessas crianças, segundo a avaliação pela escala de Gesell foi de $44,9 \%$, sendo as áreas de comportamento adaptativo $(56,8 \%)$ e de linguagem as mais comprometidas (Tabela 1).

$\mathrm{Na}$ Tabela 2 são apresentadas as principais características socioeconômicas e demográficas das famílias estudadas. Configura-se uma situação de condições socioeconômicas ruins demonstradas por mais de $50 \%$ de famílias com renda menor ou igual a 1 salário mínimo, apenas 5,1\% de mães com mais de 8 anos de escolaridade e um desemprego paterno de $27,3 \%$ ou mais. Além disso, apenas $67,1 \%$ das mães fizeram mais de 4 consultas de pré natal. As condições de moradia e saneamento também apontam para dificuldades diárias visto que $100 \%$ das famílias não contavam com água encanada e 69,9\% com sanitário em sua residência. A pesar de não contarem, em sua maioria, com acesso aos serviços públicos, bens de consumo duráveis como geladeira, televisão e fogão estavam presentes em mais de $70 \%$ das residências.
A presença de estímulos favoráveis ao desenvolvimento infantil, medidos segundo o Inventory Home nos ambientes domésticos, foi baixa, especialmente daquel es ligados à organização do ambiente (baixo em $64,2 \%$ dos domicílios) e à presença de materiais de aprendizagem (baixo em 73,9\% dos domicílios) com se percebena Tabela 3 .

$\mathrm{Na}$ análise de fatores de risco socioeconômicos para baixos escores do Inventory $\mathrm{H}$ ome, tanto na análise não ajustada como na ajustada, a pobreza extrema (renda familiar menor que 1 salário mínimo), baixas escolaridades materna e paterna ( $<5$ anos), número de pessoas residentes no domicílio $(\geq 5)$ enúmero defilhos menores que 5 anos ( $\geq 2)$ mostraram associação significante. 0 número de cômodos do domicílio $(<4)$ associou-se apenas na análise não ajustada (Tabela 4).

Foram feitas duas análises de fatores de risco para suspeitas de atraso no desenvolvimento, medidas pela escala de Gesell (tabela 5). Na primeira, procedeu-seàsanálisesnão ajustada eajustada sem a inclusão da avaliação do Inventory Home total de cada família no modelo. Nesse momento, associaram-se à suspeita de atraso no desenvolvimento a pobreza extrema (renda familiar <1 salário mínimo), baixa escolaridade

Tabela 1. Desenvolvimento de crianças de 2 anos de idade da Vila Cidade Olímpica segundo a Escala de Gesell, no município de São Luís, MA - 2003.

\begin{tabular}{|c|c|c|}
\hline $\begin{array}{c}\text { Áreas do comportamento } \\
\text { segundo Gesell }\end{array}$ & $f$ & $\%$ \\
\hline \multicolumn{3}{|l|}{ Comportamento Adaptativo } \\
\hline Normal & 76 & 43,2 \\
\hline Suspeita de atraso & 100 & 56,8 \\
\hline \multicolumn{3}{|l|}{ Comportamento M otor Grosso } \\
\hline Normal & 176 & 100 \\
\hline Suspeita de atraso & - & \\
\hline \multicolumn{3}{|l|}{ Comportamento M otor Fino } \\
\hline Normal & 109 & 61,9 \\
\hline Suspeita de atraso & 67 & 38,1 \\
\hline \multicolumn{3}{|l|}{ Comportamento de Linguagem } \\
\hline Normal & 65 & 36,9 \\
\hline Suspeita de atraso & 111 & 63,1 \\
\hline \multicolumn{3}{|l|}{ Comportamento Pessoal e Social } \\
\hline Normal & 134 & 76,1 \\
\hline Suspeita de atraso & 42 & 23,9 \\
\hline \multicolumn{3}{|l|}{ Gesell total } \\
\hline Normal & 97 & 55,1 \\
\hline Suspeita de atraso & 79 & 44,9 \\
\hline
\end{tabular}


Tabela 2. Análise das tendências dos indicadores das condições de saúde e do uso de serviços de saúde entre idosos brasileiros (PNAD, 1998, 2003, 2008)

\begin{tabular}{|c|c|c|c|c|c|}
\hline Varáveis socioeconômicas & $f$ & $\%$ & Variáveis demográficas & $f$ & $\%$ \\
\hline Renda familiar (SM) & & & Tipo de construção & & \\
\hline$<1$ SM & 82 & 46,6 & Alvenaria & 136 & 77,3 \\
\hline $1 \mathrm{SM}$ & 45 & 25,6 & Taipa & 40 & 22,7 \\
\hline$>1 \mathrm{SM}$ & 49 & 27,8 & № de cômodos & & \\
\hline Escolaridade materna & & & $1-2$ & 71 & 40,4 \\
\hline $0-4$ & 90 & 51,1 & 3 ou mais & 105 & 59,6 \\
\hline $5-8$ & 77 & 43,8 & Água (rede pública) & & \\
\hline$>8$ & 9 & 5,1 & Sim & - & - \\
\hline O cupação paterna & & & Não & 176 & 100 \\
\hline Trabalhando & 94 & 53,4 & Banheiro com sanitário & & \\
\hline Desempregado & 48 & 27,3 & Sim & 53 & 30,1 \\
\hline Não sabe informar & 34 & 19,3 & Não & 123 & 69,9 \\
\hline Idade materna (anos) & & & Coleta publica de lixo & & \\
\hline $16-19$ & 17 & 9,7 & Sim & 140 & 79,5 \\
\hline $20-29$ & 122 & 69,3 & Não & 36 & 20,5 \\
\hline 30 a 40 & 37 & 21,0 & Rádio & & \\
\hline № de filhos $<$ de 5 anos & & & Sim & 102 & 58,0 \\
\hline 1 filho & 66 & 37,5 & Não & 74 & 42,0 \\
\hline 2 ou mais & 110 & 62,5 & Televisão & & \\
\hline № de pessoas na família & & & Sim & 143 & 81,2 \\
\hline $3-4$ pessoas & 94 & 53,4 & Não & 33 & 18,8 \\
\hline 5 ou mais & 82 & 46,6 & & & \\
\hline
\end{tabular}

Tabela 3. Caracterização do ambiente doméstico segundo a classificação do Inventory HOME, na Vila Cidade Olímpica, no município de São Luís, MA - 2003.

\begin{tabular}{|c|c|c|}
\hline \multicolumn{3}{|c|}{ Escalas do Inventory HOME } \\
\hline Elementos avaliados & $f$ & $\%$ \\
\hline \multicolumn{3}{|l|}{ Responsividade } \\
\hline Baixo & 96 & 54,6 \\
\hline M édio & 72 & 40,9 \\
\hline Alto & 8 & 4,5 \\
\hline \multicolumn{3}{|l|}{ Aceitação } \\
\hline Baixo & 91 & 51,7 \\
\hline M édio & 82 & 46,6 \\
\hline Alto & 3 & 1,7 \\
\hline \multicolumn{3}{|l|}{ Organização do ambiente } \\
\hline Baixo & 113 & 64,2 \\
\hline M édio & 62 & 35,2 \\
\hline Alto & 1 & 0,6 \\
\hline \multicolumn{3}{|l|}{ M ateriais de aprendizagem } \\
\hline Baixo & 130 & 73,9 \\
\hline M édio & 44 & 25,0 \\
\hline Alto & 2 & 1,1 \\
\hline \multicolumn{3}{|l|}{ Envolvimento } \\
\hline Baixo & 98 & 55,7 \\
\hline M édio & 68 & 38,6 \\
\hline Alto & 10 & 5,7 \\
\hline \multicolumn{3}{|l|}{ Variedade de estímulos } \\
\hline Baixo & 85 & 48,3 \\
\hline M édio & 86 & 48,9 \\
\hline alto & 5 & 2,8 \\
\hline \multicolumn{3}{|l|}{ Home Total } \\
\hline baixo & 100 & 56,8 \\
\hline médio & 69 & 39,2 \\
\hline alto & 7 & 4,0 \\
\hline
\end{tabular}

materna ( $\leq 5$ anos), mais de 2 filhos $<5$ anos e mais de 4 pessoas residentes no domicílio. Ao introduzir-se a variável escore HOME total baixo no modelo ajustado mantiveram-se como fatores de risco apenas o próprio HOME total baixo, a presença de mais de 2 filhos $<5$ anos e mais de 4 pessoas residentes no domicílio.

\section{Discussão}

As condições socioeconômicas das famílias estudadas se mostraram extremamente desfavoráveis com 46,59\% delas vivendo com menos de 1 salário mínimo (menos de 100 dólares americanos, na época da coleta), escolaridades materna e paterna muito baixas, com desemprego paterno alto e com pouco acesso a serviços públicos. Essa situação é característica de periferias das grandes cidades brasileiras e tende a gerar ambientes com poucos estímulos favoráveis ao de senvolvimento normal das crianças que ali residem $4,5,8,15$. A provável consequência dessa situação se confirma quando verificamos um baixo escore total do Inventory HOM E em $56,8 \%$ desses domicílios e apenas 4\% de residências estudadas com estímulos adequados. 
Tabela 4. Análises não ajustada e ajustada dos fatores demográficos e socioeconômicos em relação ao Escore Home total no ambiente doméstico de crianças de 2 anos de idade, na Vila Cidade Olímpica, no município de São Luís, 2003.

\begin{tabular}{|c|c|c|c|c|c|c|c|c|}
\hline \multirow[b]{3}{*}{ Variáveis } & \multicolumn{5}{|c|}{ Análise não ajustada } & \multicolumn{3}{|c|}{ Análise ajustada } \\
\hline & \multicolumn{2}{|c|}{ HOME Baixo } & \multirow[b]{2}{*}{ OR } & \multirow[b]{2}{*}{ IC 95\% } & \multirow[b]{2}{*}{$p$} & \multirow[b]{2}{*}{ OR } & \multirow[b]{2}{*}{ IC 95\% } & \multirow[b]{2}{*}{$p$} \\
\hline & $f$ & $\%$ & & & & & & \\
\hline \multicolumn{9}{|c|}{ Renda familiar (SM) } \\
\hline$<1$ & 64 & 78,0 & 5,7 & $2,80-11,85$ & 0,0000 & 5,9 & $2,0-17,1$ & 0,001 \\
\hline 1 ou mais & 36 & 38,3 & & & & & & \\
\hline \multicolumn{9}{|l|}{ Escolaridade (mãe) } \\
\hline $0-4$ anos & 80 & 88,9 & 26,40 & $10,81-66,29$ & 0,0000 & 42,7 & $13,6-134$ & $<0,001$ \\
\hline 5 anos ou mais & 20 & 23,3 & & & & & & \\
\hline \multicolumn{9}{|l|}{ Escolaridade (pai) } \\
\hline $0-4$ anos & 77 & 66,4 & 3,18 & $1,58-6,40$ & 0,0003 & 3,0 & $1,0-8,31$ & 0,03 \\
\hline 5 anos ou mais & 23 & 38,3 & & & & & & \\
\hline \multicolumn{9}{|c|}{ № de pessoas em casa } \\
\hline 5 ou mais & 55 & 67,1 & 2,22 & $1,15-4,30$ & 0,01 & 4,5 & $1,5-13,0$ & 0,005 \\
\hline $3-4$ & 45 & 47,9 & & & & & & \\
\hline \multicolumn{9}{|c|}{ № de filhos < de 5 anos } \\
\hline 2 ou mais & 75 & 68,2 & 3,51 & $1,77-7,02$ & $<0,001$ & 4,6 & $1,5-13,7$ & 0,005 \\
\hline 1 & 25 & 37,9 & & & & & & \\
\hline \multicolumn{9}{|l|}{ № de cômodos } \\
\hline $1-3$ & 70 & 67,3 & 2,88 & $1,48-5,64$ & $<0,001$ & 2,2 & $0,7-6,6$ & 0,14 \\
\hline 4 ou mais & 30 & 41,7 & & & & & & \\
\hline
\end{tabular}

Tabela 5. Análises não ajustada e ajustada dos fatores demográficos e socioeconômicos em relação à avaliação de Gesell

\begin{tabular}{|c|c|c|c|c|c|c|c|c|}
\hline \multirow[b]{3}{*}{ Variáveis } & \multicolumn{5}{|c|}{ Análise não ajustada } & & & \\
\hline & \multicolumn{2}{|c|}{$\begin{array}{c}\text { Com suspeita } \\
\text { de atraso }\end{array}$} & \multirow[b]{2}{*}{ OR } & \multirow[b]{2}{*}{ IC $95 \%$} & \multirow[b]{2}{*}{$p$} & \multicolumn{3}{|c|}{ Análise ajustada (s/ HOME) } \\
\hline & $f$ & $\%$ & & & & OR & IC $95 \%$ & $p$ \\
\hline \multicolumn{9}{|l|}{ Renda familiar (SM) } \\
\hline$<1$ & 46 & 56,1 & 2,36 & $1,23-4,55$ & 0,05 & 2,0 & $0,9-4,0$ & 0,052 \\
\hline \multirow{2}{*}{\multicolumn{9}{|c|}{ Escolaridade da mãe }} \\
\hline & & & & & & & & \\
\hline $0-4$ anos & 53 & 58,9 & 3,31 & $1,69-6,48$ & 0,001 & 3,1 & $1,5-6,4$ & 0,002 \\
\hline 5 anos ou mais & 26 & 30,2 & & & & & & \\
\hline \multicolumn{9}{|l|}{ № de filhos $<$ de 5 anos } \\
\hline 2 ou mais & 61 & 55,5 & 3,32 & $1,61-6,79$ & $<0,001$ & 2,7 & $1,3-5,7$ & 0,006 \\
\hline 1 filho & 18 & 27,3 & & & & & & \\
\hline \multicolumn{9}{|l|}{ № de pessoas em casa } \\
\hline 5 ou mais pessoas & 51 & 62,2 & 3,88 & $1,98-7,65$ & $<0,001$ & 4,5 & $2,2-9,3$ & $<0,001$ \\
\hline \multirow[t]{3}{*}{$3-4$ pessoas } & 28 & 29,8 & & & & & & \\
\hline & & & & & & \multicolumn{3}{|c|}{$\begin{array}{l}\text { Análise ajustada } \\
\text { (incluindo HOME) }\end{array}$} \\
\hline & & & & & & OR & IC $95 \%$ & $\mathrm{p}$ \\
\hline Renda familiar & & & & & & 1,5 & $0,7-3,2$ & 0,27 \\
\hline Escolaridade da mãe & & & & & & 1,5 & $0,6-3,8$ & 0,36 \\
\hline № de filhos $<5$ anos & & & & & & 2,3 & $1,1-4,9$ & 0,02 \\
\hline № de pessoas em casa & & & & & & 3,7 & $1,8-7,7$ & $<0,001$ \\
\hline HOME Total baixo & & & & & & 3,1 & $1,1-8,4$ & 0,02 \\
\hline
\end{tabular}


$\mathrm{Na}$ investigação dos fatores envolvidos na determinação desse ambiente inadequado para o desenvolvimento infantil, constata-se quetanto a renda familiar muito baixa quanto a baixa escolaridade dos pais seriam fatores independentes. Porém, chama a atenção a associação, na análise ajustada, de variáveis demográficas como o número de pessoas residentes e o número de crianças com menos de 5 anos deidade presentes na mesma casa. É plausível pensar que crianças pequenas tenham que dividir os poucos brinquedos e a atenção dos pais com irmãos ou primos mais velhos, reduzindo suas chances de obter estímulos benéficos. Isso corrobora tanto 0 trabalho de $\mathrm{H}$ al pern et al. ${ }^{7}$, o qual constatou que em famílias onde existiam quatro ou mais irmãos a suspeita para atrasos no desenvolvimento era de $90 \%$, como o de $\mathrm{M}$ artins et al. ${ }^{16}$, que encontrou oito fatores de risco associados à baixa qualidade do ambiente: baixa renda familiar mensal, baixa escolaridade materna, sexo masculino, casas com mais de sete residentes, número de irmãos maior ou igual a quatro, uso de tabaco na gestação, crianças que dormem na cama dos pais aos 4 anos e mães com presença de transtornos psiquiátricos.

Reforçando a ideia de que 0 ambiente doméstico pobre em estímulos concorre para re duzir as chances de um desenvolvimento infantil adequado, verificou-se numa primeira análise que a renda familiar e a escolaridade materna, além das variáveis demográficas citadas acima, se associaram com suspeitas de atraso no desenvolvimento das crianças estudadas, observadas no teste de Gesell. Fica clara, porém, a importância individual dessas duas variáveis ( $\mathrm{n}$ - de crianças $<5$ anos e $n$ o de pessoas residentes no domicílio) quando acrescentamos ao modelo a variável HOM E total, ou seja, uma medida dos estímulos ambientais domésticos. N essemomento, deixam de se associar renda familiar e escolaridade materna, mas mantém-se a associação do HOME total edas variáveis no de crianças $<5$ anos emais de 4 pessoas residentes no domicílio.

A influência deum número exagerado depessoas adultas ou crianças residindo no mesmo domicílio foi bem documentada em trabalhos como os de Andrade et al. ${ }^{8}$, Guerra ${ }^{17}$, Eickmann et al. ${ }^{18}$ e Lima et al. ${ }^{19} \mathrm{em}$ periferia de Recife, capital do estado de Pernambuco, nordeste do Brasil, e Halpern ${ }^{7}$ em Pelotas, Rio Grande do Sul, no sul do Brasil. Porém, diferentemente do presentetrabal ho, em todas essas pesquisas outras variáveis socioeconômicas como renda familiar e escolaridade da mãe continuaram se associando com suspeitas de atrasos no desenvolvimento, mesmo nas análises multivariadas.

Os resultados dessa pesquisa mostram que o ambiente doméstico é fundamental para o desenvolvimento de crianças na faixa de dois anos de idade e, provavelmente, também para crianças de outras faixas etárias. Pode-se também apontar elementos com maior contribuição na gênese de suspeitas de atraso no desenvolvimento infantil tais como a presença de hiperlotação na casa, tanto de adultos como de crianças menores de 5 anos, além da baixa intensidade de estímulos favoráveis verificada nas mesmas.

\section{Colaboradores}

F Lamy Filho, SM M edeiros, ZC Lamy e M EL $M$ oreira participaram igualmentedetodas as etapas da elaboração do artigo. 


\section{Referências}

1. Lordelo ER, Fonseca AL, Araújo, M LVB. Responsividade do ambiente de desenvolvimento: crenças e práticas como sistema cultural de criação de filhos Psicol. Reflex. Crit 2000; 13(1):73-80.

2. Petrariu FD, Gavãt V, Amarandei ME. Assessment of the impact of psycho-social environment on toddlers' physical and neuropsychic development Rev Med Chir Soc M ed Nat Iasi. 2007; 111(4):1028-1034.

3. Brazelton TB. As necessidades essenciais da criança: 0 que toda criança precisa para crescer, aprender e se desenvolver. Porto Alegre: Artmed; 2002.

4. Huston AC, M cloyd VC, Coll CG. Children and poverty: issues in contemporary research. Child Dev 1994; 65(2):275-282.

5. Sameroff AJ. Systems, development, and early intervention. M onogr Soc Res Child Dev 1992; 57(6):154163.

6. Bradley RH, Corwyn RF. Socioeconomic status and child development. Annu rev Psychol 2002; 53:371399.

7. Halpern R, Giugliani, ERJ, Victora CG, Barros FC, Horta BL. Fatores de risco para suspeita de atraso no desenvolvimento neuropsicomotor aos 12 meses de vida I Pediatric 2000; 76(6):421-428.

8. Andrade AS, Santos DN, Bastos AC, Pedromônico MRM, Almeida-Filho N, Barreto M L. Ambiente familiar e desenvolvimento cognitivo infantil: uma abordagem epidemiológica. Rev Saúde Pública 2005; 39(4):606-611.

9. Cappellini AC, Mancini S, Zuffellato S, Bini F, Polcaro $\mathrm{P}$, Conti AA, Molino Lova R, Macchi C. Environmental effects on school age child psychomotricity. M inerva Pediatr. 2008; 60(3):277-284.

10. Fluss J, Ziegler J, Ecalle J, Magnan A, Warszawski J, Ducot B, Richard G, Billard C. Prevalence of reading disabilities in early elementary school: I mpact of socioeconomic environment on reading development in 3 different educational zones. Arch Pediatr. 2008; 14(6):1049-1057.

11. Secretaria M unicipal de Saúde de São Luís. Anuário 2002. São Luís: SEM US; 2002.

12. Piaget J. A linguagem e o pensamento da criança. 6ạ. ed. São Paulo: M artins Fontes; 1993.
13. Bradley RH, Caldwell BM. HOME Inventory Manual Administration M anual. 3a ed. Little Rock: University of Arkansas at Little Rock; 2001.

14. Gesell A. A criança de 0 a 5 anos. 5a ed. São Paulo: M artins Fontes; 1999.

15. Bastos ACS, Urpia ACM, Pinho L, Almeida Filho NM. O impacto do ambiente familiar nos primeiros anos de vida: um estudo com adolescentes de uma invasão de Salvador, Bahia. Estud. Psicol 1999; 4(2):239-271.

16. Martins M FD, Costa JSD, Saforcada ET, Cunha MDC. Qualidade do ambiente e fatores associados: um estudo em crianças de Pelotas, Rio Grande do Sul, Brasil. Cad. Saúde Pública 2004; 20(3):710-718.

17. Guerra MQ. Fatores associados à qualidade de estimulação do ambiente doméstico de crianças aos 12 meses de idade na zona da mata de Pernambuco [dissertação]. Recife (PE): Universidade Federal de Pernambuco; 2002.

18. Eickmann SH, Lima MC, Guerra MQ, , Lira PIC, Sharon R A, Huttly SRA, Ashworth A. Improved cognitive and motor development in a communitybased intervention of psychosocial stimulation in northeast Brazil. Developmental Medicine \& Child N eurology 2003;45(8):536-541.

19. Lima M C, Eickmann SH, Lima ACV, Guerra M Q, Lira PIC, Huttly SRA, Ashworth A. Determinants of mental and motor development at 12 months in a low income population: a cohort study in northeast Brazil. Acta Paediatrica 2004; 93(7):969-975.

Artigo apresentado em 16/06/2008

Aprovado em 05/09/2008

Versão final apresentada em 19/09/2008 